\title{
Predictores de la percepción de riesgo y del comportamiento ante el cambio climático. Un estudio piloto
}

\author{
M. Carmen Hidalgo e Ignacio Pisano \\ Universidad de Málaga
}

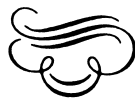

Resumen

Diferentes estudios han mostrado como determinantes de la percepción de riesgo del cambio climático diversas variables tales como las actitudes hacia el medio ambiente, los conocimientos sobre el cambio climático o la autoeficacia (Heath y Gifford, 2006; Sundblad, Biel y Gärling, 2007). Sin embargo, la falta de consenso en estos trabajos, así como la ausencia de escalas de actitudes específicas hacia el cambio climático sugieren la necesidad de profundizar en esta línea. En este estudio piloto se analizan conjuntamente los predictores de la percepción de riesgo y de la disposición a actuar contra el cambio climático más relevantes hallados en la literatura previa. Para ello se ha elaborado una escala tipo Likert para evaluar las variables objeto de estudio: conocimiento, actitudes hacia el cambio climático y autoeficacia como variables predictoras y percepción de riesgo e intención de conducta como variables criterio. Esta escala ha sido puesta a prueba en una muestra de 84 estudiantes universitarios. La consistencia interna de las escalas son aceptables, excepto la variable actitud hacia el cambio climático que obtiene un nivel bajo. Los resultados de los análisis de regresión indican que la percepción sobre la realidad del cambio climático se explica, en un $44 \%$ de la varianza, a partir del nivel de conocimientos, la actitud hacia el cambio climático y la autoeficacia. La intención de conducta se predice en un $17 \%$ a partir de la actitud hacia el cambio climático y la autoeficacia.

Palabras clave: Percepción de riesgo, cambio climático, comportamiento proambiental.

\section{Determinants of risk perception and willingness to tackle climate change. A pilot study}

\begin{abstract}
Different research papers have demonstrated that risk perception related to climate change can be attributed to different variables such as general environmental beliefs, knowledge about climate change and self-efficacy (Heath and Gifford, 2006; Sundblad, Biel and Gärling, 2007). However, the lack of agreement in the work carried out so far and the need to define specific scales of attitudes towards climate change indicate that further research must be conducted along these lines. This pilot study analyzes the determinants of risk perception and behavioral intentions in relation to climate change found in the literature. A Likert-type scale was developed to assess the following variables: knowledge, attitudes towards climate change and self-efficacy as predictive variables and risk perception and behavioral intentions as criteria variables. This scale was tested on 84 university students. The internal consistency of the scale was acceptable for all variables with the exception of attitude toward climate change where consistency was low. The results of the regression analyses showed that $44 \%$ of the variance observed in risk perception related to climate change can be explained by the level of knowledge, attitudes toward climate change and self-efficacy. $17 \%$ of the variance observed in relation to behavioral intentions can be predicted by attitudes toward climate change and self-efficacy.
\end{abstract}

Keywords: Risk perception, climate change, pro-environmental behavior.

Correspondencia con los autores: Departamento de Psicología Social, Ant. Social, Trabajo Social y S.S. Facultad de Psicología. Universidad de Málaga. Campus de Teatinos, s/n. 29071 Málaga. E-mails: mchidalgo@uma.es ignaciopisano@gmail.com 


\section{Introducción}

En la actualidad, el cambio climático se ha posicionado como el principal problema ambiental que manifiestan los ciudadanos (Kull, 2007; Moyano, Jiménez y Lafuente, 2007; Special Eurobarometer 295, 2008). Por otra parte, la comunidad científica encargada de analizar y debatir el problema del cambio climático desde hace años además de confirmar que nos encontramos ante uno de los retos más grandes que debe afrontar la humanidad, ha reiterado una y otra vez que el aumento de la temperatura experimentado en el último siglo se debe al incremento de gases de efecto invernadero emitidos a la atmósfera principalmente por la actividad humana (Intergovernmental Panel on Climate Change, 2007). Lo que es peor aún, entre sus consecuencias se encuentran sequías e inundaciones, olas de calor, aumento del nivel del mar, problemas de salud, disminución de flora y fauna, etc. En este escenario, por lo tanto, el cambio climático es concebido ya no sólo como un problema de las generaciones futuras sino como un riesgo inminente en las actuales sociedades.

Desde el campo de las ciencias sociales y particularmente desde la psicología ambiental, se ha visto incrementado el número de estudios que intentan conocer la percepción social de este riesgo, los conocimientos, actitudes y creencias específicas ante este problema y, quizás lo más importante, el abordaje de las posibles acciones individuales y colectivas para hacerle frente (Brody, Zahran, Vedlitz y Glover, 2008; Heath y Gifford, 2006; Sundblad, Biel y Gärling, 2007; Whitmarsh, 2009). En el presente estudio se analiza la percepción de riesgo sobre la realidad del cambio climático, la disposición a actuar contra éste y, en especial, los predictores de ambas variables.

Entre los factores que han demostrado tener una cierta relación predictiva con la percepción de riesgo destacan el nivel de conocimientos sobre el cambio climático, las actitudes generales hacia el medio ambiente así como la percepción de autoeficacia. Así, de acuerdo con diferentes autores (Bord, O'Connor y Fisher, 2000; O'Connor, Bord y Fisher, 1999; Sundblad et al., 2007), el conocimiento de las causas y de las consecuencias que tendrá el cambio climático aumenta la consiguiente percepción de riesgo de este fenómeno, así como la disposición a actuar para hacerle frente. Sin embargo, en un estudio reciente de Brody et al. (2008) el nivel de conocimientos no resulta significativo en la predicción de la percepción de riesgo, por lo que, como estos mismos autores señalan, son necesarios nuevos estudios que ayuden a clarificar dicha relación.

En cuanto a las actitudes generales hacia el medio ambiente, Heath y Gifford (2006), utilizando la escala de Thomson y Barton (1994) encuentran que el ecocentrismo es un buen predictor de la percepción de riesgo de que el cambio climático esté ocurriendo y de la intención de actuar contra éste, mientras que la apatía medioambiental se considera un buen predictor, pero en sentido negativo, de ambas variables. El trabajo de Brody et al. (2008), esta vez utilizando la escala del Nuevo Paradigma Ecológico (NEP) de Dunlap, van Liere, Mertig y Jones (2000), confirma este efecto de las actitudes ambientales sobre la percepción de riesgo. Otros trabajos señalan igualmente la relación predictiva entre actitudes proambientales e intención de conducta (Böhm y Pfister, 2001; O'Connor et al., 1999). Así, O'Connor et al. (1999), utilizando una escala de actitudes generales hacia el medio ambiente, encontraron que actitudes más proambientales se relacionan con una mayor intención de actuar contra el cambio climático (por ejemplo usando menos aire acondicionado en verano y menos calefacción en invierno o votando medidas políticas para mitigar las causas del cambio climático). En otro estudio similar Bord, Fisher y O’Connor (1998) encontraron que las personas que mantienen la creencia de que el mundo es "frágil" tienen más probabilidad de adoptar conductas y apoyar políticas que mitiguen el riesgo asociado al cambio climático. No obstante, hasta el momento no se han utilizado escalas específicas de actitudes hacia el cambio climático, a pesar de que, tanto los estudios clásicos sobre actitudes (Fishbein y Ajzen, 1975) como 
otros de este campo (e.g. Stern, 2000) abogan por una mayor correspondencia entre el nivel de medida de la actitud y el de la conducta.

Asimismo, algunas investigaciones demuestran que las personas que se autoperciben como capaces de afectar positivamente al cambio climático, como así también de influenciar a otros de su entorno social más cercano, poseen una probabilidad más alta de definir el cambio climático como un riesgo y de llevar a cabo acciones correctoras. De hecho, en el estudio de Brody et al. (2008) la autoeficacia aparece como la variable más significativa del modelo predictivo sobre la percepción de riesgo, mientras que otros trabajos (Bord et al., 1998; Heath y Gifford, 2006; O’Connor et al., 1999; Savage, 1993) la señalan como un factor significativo de la intención de actuar. Por lo tanto, es de esperar que la autoeficacia sea un prerrequisito tanto de cómo es percibido el cambio climático como de los esfuerzos personales para combatirlo.

La propia percepción de riesgo sobre el cambio climático, a su vez, ha sido asociada a la disposición a llevar a cabo acciones individuales para mitigar los efectos sobre el medio ambiente de este fenómeno. Diversos trabajos han mostrado que la percepción de riesgo es un buen predictor de la intención de conducta para hacer frente al cambio climático (Heath y Gifford, 2006; O'Connor et al., 1999; O’Connor, Bord, Yarnal y Wiefek, 2002). O'Connor et al. (2002) demuestran cómo aquellos sujetos que perciben el cambio climático como una situación de riesgo sustancial apoyan en mayor grado iniciativas políticas que supongan un cambio en el modelo energético como así también ciertas acciones voluntarias como pueden ser comprar productos "verdes", conducir menos o escoger aquellas compañías proveedoras de energía que son consideradas menos contaminantes.

De acuerdo con la revisión realizada, nos proponemos analizar conjuntamente las variables que han resultado significativas en diferentes estudios previos sobre la percepción de riesgo del cambio climático y sobre la disposición a actuar para hacerle frente. Concretamente esperamos encontrar un efecto significativo del nivel de conocimientos sobre las causas de este fenómeno medioambiental, de las actitudes específicas hacia el cambio climático y de la autoeficacia sobre la percepción de riesgo así como de estas cuatro variables (percepción de riesgo, actitudes, conocimientos y autoeficacia) sobre la disposición a actuar.

\section{Método}

\section{Participantes}

La muestra se compone de 84 alumnos de las Facultades de Ciencias de la Comunicación y Educación; 24 varones y 60 mujeres, con edades comprendidas entre los 18 y 49 años siendo la media de 24 años $(\mathrm{S} x=4,8)$.

\section{Instrumento}

Para realizar esta investigación se elaboró un cuestionario a partir de ítems empleados en estudios previos de diferentes autores. En él se recogen aspectos socio-demográficos (edad, sexo, estado civil e ideología política) así como las variables analizadas en este estudio, operacionalizadas en 19 ítems con una escala tipo Likert de 7 puntos ( $1=$ totalmente en desacuerdo a 7 = totalmente de acuerdo):

Conocimiento sobre las causas del cambio climático: compuesto por 5 ítems que hacen referencia básicamente a las causas antropogénicas, naturales o de los gases de efecto invernadero que producen el cambio climático. Estos ítems han sido extraídos de Heath y Gifford (2006) y de Sundblad et al. (2007).

Actitud hacia el cambio climático: incluye 3 ítems de elaboración propia que recogen aspectos relacionados con la preocupación por el cambio climático actual, las sanciones por el incumplimiento del Protocolo de Kioto o el posicionamiento hacia las amenazas medioambientales. 
Autoeficacia: compuesto por 2 ítems "Existen simples cosas que puedo hacer que tendrían un efecto significativo para aliviar las consecuencias negativas del cambio climático" y "Es muy difícil que alguien como yo pueda hacer algo por el medio ambiente". Extraídos de Centro de Investigaciones Sociológicas (2004) y de Heath y Gifford (2006).

Percepción de riesgo: incluye 8 ítems que hacen referencia a dos aspectos que son: percepción de que el cambio climático está ocurriendo (5 ítems) y la percepción de las consecuencias que este fenómeno puede tener ( 3 ítems). Extraídos de Heath y Gifford (2006) y de Sundblad et al. (2007).

Intención de conducta: compuesto por el ítem "Estaría dispuesto a introducir cambios en mi modo de vida para combatir el cambio climático”.

\section{Procedimiento}

El cuestionario fue aplicado de forma colectiva en un aula de cada Centro (Ciencias de la Comunicación y Educación) en horario de clases. La duración media para su realización fue de 20 minutos. La participación de los sujetos y la cumplimentación del cuestionario fue anónima, voluntaria y gratuita.

\section{Resultados}

Como puede observarse en la tabla I las puntuaciones medias de las seis variables son considerablemente altas. De ellas destaca la percepción de riesgo $(M=5,69)$, la autoeficacia $(M=5,80$. $)$ y la intención de conducta $(M=5,95)$, todas en una escala de 7 puntos. La consistencia interna de las escalas son aceptables, excepto la variable actitud que obtiene un $\alpha=0.43$. Con el propósito de analizar en mayor detalle la fiabilidad de esta última escala se realizó un análisis de la capacidad de discriminación de los ítems (Likert, 1932). Como puede verse en la tabla II los ítems de dicha escala poseen una adecuada capacidad de discriminación.

TABLA I

Estadísticos descriptivos y Fiabilidad de las variables analizadas

\begin{tabular}{lrrrrr}
\hline & Media & $\begin{array}{c}\text { Desviación } \\
\text { típica }\end{array}$ & Mínimo & Máximo & Alpha \\
\hline Conocimiento & 5,18 &, 80 & 3,00 & 7,00 & .60 \\
Actitud & 5,33 &, 93 & 2,87 & 7,00 & .43 \\
Autoeficacia & 5,80 & 1,33 & 1,00 & 7,00 & .79 \\
Percepción de Riesgo & 5,69 &, 83 & 3,57 & 7,00 & .75 \\
Intención Conducta & 5,95 & 1,10 & 2,00 & 7,00 & \\
\hline
\end{tabular}

TABLA II

Prueba T de Likert

\begin{tabular}{ccc}
\hline \multirow{2}{*}{ Percepción de riesgo } & \multicolumn{2}{c}{ Prueba T de Likert } \\
\cline { 2 - 3 } & $t$ & Sig. \\
\hline Ítem 1 & $-8,88$ & .000 \\
Ítem 2 & $-4,39$ & .000 \\
Ítem 3 & $-5,97$ & .000 \\
\hline
\end{tabular}

La tabla III muestra las correlaciones entre las variables. La mayoría de las relaciones hipotetizadas se han confirmado. Así, la percepción de riesgo correlaciona positivamen- 
te con el conocimiento, la actitud, la autoeficacia y la intención de conducta. Ésta última se relaciona positivamente con la actitud, la autoeficacia y la percepción de riesgo, no resultando significativa la relación con el nivel de conocimientos.

TABLA III

Correlaciones entre las variables

\begin{tabular}{lrrrrr}
\hline & Conocimiento & Actitud & Autoeficacia & Pp de Riesgo & $\begin{array}{r}\text { Intención } \\
\text { Conducta }\end{array}$ \\
\hline Conocimiento & 1 &, $454 * *$ &, $303 * *$ &, $475 * *$ &, 206 \\
Actitud & & 1 &, $582^{* *}$ &, $589 * *$ &, $317 * *$ \\
Autoeficacia & & & 1 &, $547 * *$ &, $390^{* *}$ \\
Ppde Riesgo & & & & 1 &, $382^{* *}$ \\
Intención Cta & & & & & 1 \\
\hline
\end{tabular}

$* * p<0,01$

Por último, con el objetivo de determinar los diferentes antecedentes de la percepción de riesgo y de la intención de conducta en relación al cambio climático, se realizaron dos análisis de regresión (véase Tablas IV y V). El primero de ellos resultó estadísticamente significativo $(F(3,80)=22,59 ; p<0,001)$ explicando un $44 \%$ de la varianza. Como puede verse en la tabla IV, los tres predictores influyen significativamente en el factor percepción de riesgo, siendo la actitud y la autoeficacia los que presentan valores similares y superiores en el coeficiente beta. En cuanto a la intención de conducta como variable dependiente, los resultados obtenidos mostraron que dos de los predictores resultaron estadísticamente significativos, a saber, la autoeficacia y la percepción de riesgo $(F(2,81)=9,66 ; p<0,001)$ explicando un $17 \%$ de la varianza. Quedaron fuera de la ecuación de regresión el conocimiento y las actitudes hacia el medio ambiente y hacia el cambio climático (Tabla IV).

TABLA IV

Análisis de regresión para la Percepción de Riesgo

\begin{tabular}{lccccc}
\hline & \multicolumn{2}{c}{$\begin{array}{l}\text { Coeficientes no } \\
\text { estandarizados }\end{array}$} & $\begin{array}{c}\text { Coeficientes } \\
\text { estandarizados }\end{array}$ & & \\
\cline { 2 - 4 } Variable & $\mathrm{B}$ & Error típ. & Beta & $t$ & Sig. \\
\hline ACTITUD &, 274 &, 097 &, 305 & 2,817 &, 006 \\
CONOCIMIENTO &, 257 &, 096 &, 247 & 2,671 &, 009 \\
AUTOEFICACIA &, 185 &, 064 &, 295 & 2,910 &, 005 \\
\hline
\end{tabular}

TABLA V

Análisis de regresión para la Intención de conducta

\begin{tabular}{lccccc}
\hline & \multicolumn{2}{c}{$\begin{array}{l}\text { Coeficientes no } \\
\text { estandarizados }\end{array}$} & $\begin{array}{c}\text { Coeficientes } \\
\text { estandarizados }\end{array}$ & & \\
\cline { 2 - 4 } Variable & B & Error típ. & Beta & $t$ & Sig. \\
\hline PP RIESGO &, 316 &, 157 &, 240 & 2,013 &, 047 \\
AUTOEFICACIA &, 213 &, 098 &, 259 & 2,168 &, 033 \\
\hline
\end{tabular}

\section{Discusión}

En el presente estudio se ha analizado la percepción de riesgo de un grupo de estudiantes universitarios sobre la realidad del cambio climático y su disposición a actuar contra éste. 
El objetivo que se perseguía era por un lado, poner a prueba las escalas elaboradas y por otro, analizar de forma conjunta los predictores más significativos hallados en la literatura previa. De lo anterior, podemos apuntar las siguientes conclusiones.

En primer lugar, respecto a la fiabilidad de las escalas utilizadas, hay que destacar la baja consistencia interna de la variable actitud hacia el cambio climático $(0,43)$, si bien el análisis posterior realizado sobre la capacidad de discriminación de los ítems nos indica que poseen una buena capacidad discriminante, por lo que al menos para los análisis posteriores decidimos mantener la puntuación obtenida con dicha escala. No obstante, es evidente que para futuros trabajos es necesario mejorar esta medida específica de la actitud ambiental. El resto de escalas obtienen unas puntuaciones aceptables (conocimiento: 0,60; autoeficacia: 0,79 y percepción de riesgo: 0,75).

Por otra parte, la segunda conclusión a remarcar son los moderadamente altos valores medios obtenidos en la presente investigación. Parece ser que los estudiantes poseen correctos conocimientos sobre las causas del cambio climático; lo perciben como una amenaza real y actual; están preocupados y tienen una actitud positiva hacia el medio ambiente y hacia la lucha contra el cambio climático; y, quizás lo más importante, se creen capaces de influir positivamente sobre dicho fenómeno y, en coherencia con lo anterior, declaran estar dispuestos a llevar a cabo comportamientos proambientales capaces de mitigar los efectos negativos del cambio climático. Estos resultados coinciden con los obtenidos en otros estudios demoscópicos (Fundación BBVA, 2008; Meira, Arto y Montero, 2009; Moyano et al., 2007) en los que se destaca el buen conocimiento de la población y las actitudes positivas hacia el medio ambiente en general y hacia el cambio climático en particular. Cómo traducir estos conocimientos y actitudes positivas en acciones protectoras del medio ambiente es uno de los grandes retos al que se enfrentan las ciencias sociales en este comienzo de siglo.

En cuanto a la búsqueda de predictores, los análisis de regresión nos permiten confirmar, tal como esperábamos, similares relaciones predictivas a las encontradas en otras investigaciones que sobre ésta temática se han realizado (Brody et al., 2008; Heath y Gifford, 2006; O’Connor et al., 1999). Así, en cuanto a la percepción de riesgo, parece ser que los conocimientos sobre las causas, las actitudes específicas hacia el cambio climático y la autoeficacia percibida condicionan significativamente la percepción que se tenga de que el cambio climático supone un riesgo real e inminente. Estas tres variables explican un porcentaje significativo $(44 \%)$ de la varianza de la percepción de riesgo, lo que supone un incremento respecto a los estudios previos. A su vez, la percepción de riesgo junto a la autoeficacia se configuran como dos variables claves a la hora de explicar la intención de los sujetos para hacer frente al cambio climático de carácter antropogénico. Estos resultados nos sugiere la recomendación de tener en cuenta estas variables a la hora de diseñar programas de intervención psicoambiental que giren en torno al cambio climático. No obstante, el porcentaje de varianza explicada para la intención de conducta es modesta (17\%), quedando fuera de la ecuación de regresión los conocimientos sobre el cambio climático y las actitudes, dos variables que tal como se ha comentado en la introducción han resultado significativas en estudios anteriores (Böhm y Pfister, 2001; O’Connor et al., 1999; Sundblad et al., 2007). Una posible explicación respecto a la no relación de las actitudes específicas hacia el cambio climático puede encontrarse en la baja fiabilidad obtenida en la escala elaborada por lo que creemos que es necesario seguir profundizando en esta línea y elaborar escalas específicas hacia el cambio climático que posean buenas propiedades psicométricas. En cuanto a la variable conocimiento sobre el cambio climático, es de destacar la ausencia de correlación significativa con la intención de conducta. Al menos identificamos otro trabajo con unos resultados similares (Brody et al., 2008) pero en cualquier caso son necesarios nuevos estudios que analicen esta relación. Aquí nos hemos centrado en el nivel de conocimientos sobre las causas del cambio climático y no tanto sobre las consecuencias. Ésta sería una posible explicación a contrastar en futuras investigaciones. 
Por último, como señalan Takala (1991) y más recientemente Urbina (2006) el primer paso para abordar el problema del cambio climático debe pasar por la realización de estudios sistemáticos que logren describir y explicar cómo la gente percibe el problema, cuáles son sus conocimientos y actitudes y, sobre todo, deben aportar luz sobre las posibles acciones individuales y colectivas que sean capaces de asumir los diferentes sectores de la población. Este trabajo así como otros futuros desarrollos pretenden contribuir en dicha dirección.

\section{Referencias}

BÖHM, G. \& Pfister, H. (2001). Mental representation of global environmental risks. Research in Social Problems and Public Policy, 9, 1-30.

Bord, R., FisHer, A. \& O'CONNOR, R. (1998). Public perceptions of global warming: United States and international perspectives. Climate Research, 11, 75-84.

Bord, R., O'CONNOR, R. \& Fisher, A. (2000). In what sense does the public need to understand global climate change? Public Understanding of Science, 9 (3), 205-218.

Brody, S., Zahran, S., Vedlitz, A. \& Grover, H. (2008). Examining the Relationship Between Physical Vulnerability and Public Perceptions of Global Climate Change in the United States. Environment and Behavior, 40 (1), $72-95$.

Centro de InVESTIGACIONES SOCIOlÓGICAs (2004). Opiniones de los españoles sobre el Medio Ambiente. Estudio $\mathrm{n}^{\circ} 2557$. Febrero 2004. Centro de Investigaciones Sociológicas. Extraído el 14 de diciembre de 2008, de http://www.cis.es/cis/opencm/ES/1_encuestas/estudios/ver.jsp?estudio=3814

Dunlap, R., Van Liere, K., Mertig, A. \& Jones, R. (2000). Measuring endorsement of the New Ecological Paradigm: A revised NEP scale. Journal of Social Issues, 56 (3), 425-42.

FISHBEIN, M. \& AJZEN, I. (1975). Belief, attitude, intention and behavior: an introduction to theory and research. Reading, MA: Addison-Wesley.

FundACIÓN BBVA (2008). Percepciones y actitudes de los españoles hacia el calentamiento global. Fundación BBVA, Departamento de Estudios Sociales. Extraído el 8 de diciembre de 2008, de http://www.fbbva.es/TLFU/dat/presentacion_calentamiento_global.pdf.

HEATH, Y. \& GIFFORD, R. (2006). Free-market ideology and environmental degradation: The case of belief in global climate change. Environment and Bebavior, 38, 48-71.

Intergovernmental Panel on Climate Change (2007). 4th Assessment Report: Synthesis Report-Summary for Policy Makers. Extraido el 4 de diciembre de 2008, de http://ipcc.ch/pdf/assessment-report/ar4/ar4_syr_spm.pdf

KuLL, S. (2007). International polling on climate change. A worldpublicopinion.org analysis. Extraido el 4 de mayo de 2009 , de http://www.worldpublicopinion.org/pipa/pdf/dec07/CCDigest_Dec07_rpt.pdf

LikERT, R. (1932). A Technique for the Measurement of Attitudes. Archives of Psychology, 140, 5-53.

Meira, P., Arto, M. \& Montero, P. (2009). La sociedad ante el cambio climático. Conocimientos, valoraciones y comportamientos en la población española. Fundación Mapfre. Extraído el 10 de febrero de 2009, de http://www.mapfre.com/ccm/content/documentos/fundacion/prev-ma/cursos/la-sociedad-ante-el-cambio-climatico.pdf

MoYANO, E., JiméNEZ, M. \& LAFUENTE, R. (2007). Opinión pública y participación social sobre cuestiones ambientales. Ecobarómetro 2007. Córdoba: IESA.

O'CONNOR, R., BORD, R. \& FISHER, A. (1999). Risk perceptions, general environmental beliefs, and willingness to address climate change. Risk Analysis, 19, 461-471.

O'Connor, R., Bord, R., YARnAL, B. \& WIEFEK, N. (2002). Who wants to reduce greenhouse gas emissions? Social Science Quarterly, 83, 1-17.

Savage, T. (1993). Demographic influences on risk perceptions. Risk Analysis, 13 (4), 413-420.

SPECIAL Eurobarometer 295 (2008). Attitudes of european citizens towards the environment. Extraido el 4 de diciembre de 2008, de http://ec.europa.eu/public_opinion/archives/eb_special_en.htm

STERN, P. (2000). Toward a coherent theory of environmentally significant behaviour. Journal of Social Issues, 56 (3), $407-424$.

SundBlad, E., BIEL, A. \& GärLING, T. (2007). Cognitive and affective risk judgements related to climate change. Journal of Environmental Psychology, 27, 97-106.

TAKala, M. (1991). Environmental awareness and human activity. International Journal of Psychology, $26,585-597$.

Thomson, S. \& BARTON, M. (1994). Ecocentric and anthropocentric attitudes toward the environment. Journal of Environmental Psychology, 14, 149-157.

Urbina, J. (2006). Dimensiones psicológicas del cambio ambiental global. En J. Urbina \& J. Martínez, Más allá del cambio climático. Las dimensiones psicosociales del cambio ambiental global (pp. 65-77). México: Editorial del deporte Mexicano.

WhitmarsH, L. (2009). Behavioural responses to climate change: asymmetry of intentions and impacts. Journal of Environmental Psychology, 29, 13-23.

\section{Apéndice}

\section{Variables e ítems utilizados}

\section{Conocimiento:}

1. El cambio climático se debe a causas naturales

2. La causa principal del cambio climático es la actividad humana 
3. La emisión de gases de efecto invernadero (CO2, Metano y otros) es la principal causa del cambio climático

4. Cada vez que utilizamos carbón, petróleo o gas potenciamos el efecto invernadero

5. El principal sector causante del cambio climático es la industria (incluida la de producción energética), por delante del transporte, la ganadería y las calefacciones

\section{Actitud:}

1. Muchas de las afirmaciones sobre amenazas medio ambientales son exageradas

2. Creo que debe sancionarse de alguna manera a los países que incumplen los tratados internacionales para reducir el cambio climático, como el Protocolo de Kioto

3. En qué grado se encuentra preocupado por la situación actual del cambio climático

\section{Autoeficacia:}

1. Es muy difícil que una persona como yo pueda hacer algo por el medio ambiente

2. Existen simples cosas que puedo hacer que tendrían un efecto significativo para aliviar las consecuencias negativas del cambio climático

\section{Percepción de riesgo:}

1. Pienso que el cambio climático está ocurriendo actualmente

2. Ya he notado algunas señales del cambio climático

3. Me parece que la temperatura es más calurosa ahora que en años anteriores

4. Creo que las precipitaciones/lluvias han disminuido en los últimos años

5. La variabilidad del clima en general y los cambios bruscos e impredecibles han aumentado

6. Habrá algunas consecuencias positivas del cambio climático sobre el clima

7. Habrá algunas consecuencias positivas del cambio climático sobre el mar/glaciares

8. Habrá algunas consecuencias positivas del cambio climático sobre la salud humana

\section{Intención de conducta:}

1. Estaría dispuesto a introducir cambios en mi modo de vida para combatir el cambio climático 